\title{
Review: evidence supports surgery for lumbar disc prolapse but is insufficient for degenerative lumbar spondylosis
}

\author{
Gibson JN, Grant IC, Waddell G. The Cochrane review of surgery for lumbar disc prolapse and degenerative lumbar \\ spondylosis. Spine 1999 Sep 1;24:1820-32.
}

QUESTIONS: In patients with lumbar disc prolapse or degenerative lumbar spondylosis, is lumbar spine surgery effective, and which surgical techniques are most successful?

\section{Data sources}

Studies were identified by searching 6 databases, hand searching relevant surgical journals, scanning bibliographies of relevant articles, and contacting experts.

\section{Study selection}

Randomised controlled trials (RCTs) or quasirandomised trials on the treatment of lumbar disc prolapse or degenerative lumbar spondylosis and the associated pathological findings or clinical syndromes of back pain, instability, spinal stenosis, and degenerative spondylolisthesis were included. Studies on spinal fractures, tumours, infection, childhood deformity, steroid injections, local anaesthetic, or particular postoperative regimens were excluded.

\section{Data extraction}

Data were extracted on methods, participants, interventions, and outcomes (treatment success, need for second procedure, and spinal fusion rates).

\section{Main results}

Lumbar disc prolapse: 26 RCTs met the selection criteria. The pooled results for 5 RCTs showed that chymopapain led to greater treatment success at 3 to 12 months $\{\mathrm{p}=0.002\}^{* \dagger}$ and to less need for a second procedure at 6 to 24 months $\{\mathrm{p}<0.001\}$ *中 than did placebo (table). Heterogeneity was present among 5 RCTs that compared chemonucleolysis with standard discectomy; the combined results showed that more second procedures were done in the chymopapain group than in the surgery group $\{p<0.001\}^{*}+$ (table). A trend existed toward fewer patients with poor outcomes at 1 year in the surgery group than in the chemonucleolysis group $\{16 \%$ v $35 \%, \mathrm{p}=0.07\} *+$; this finding was statistically significant when the fixed effects model was used.

Degenerative lumbar spondylosis: no RCTs compared surgery with non-surgical treatment. 14 RCTs comparing different types of surgery met the selection criteria. Meta-analysis of 3 RCTs comparing any fusion with laminectomy showed no difference in outcomes at 18 to 24 months. The combined results of 7 heterogeneous

5364754 .
RCTs showed a trend toward a higher solid fusion rate when instrumentation was used than when no instrumentation was used. 6 of these RCTs reported clinical outcomes, and meta-analysis showed a non-significant increase in better outcomes for instrumented fusion than for graft alone. Instrumented and noninstrumented fusion did not differ for pain or disability.

\section{Conclusions}

In patients with lumbar disc prolapse, chymopapain is more effective than placebo. Discectomy may lead to better clinical outcomes with fewer second procedures than chymopapain. The effects of treatment for degenerative lumbar spondylosis are inconclusive.

*Numbers calculated from data in article.

†Gibson JN, Grant IC, Waddell G. Surgery for lumbar disc prolapse. Cochrane Review, latest version 24 Feb 1999. In: the Cochrane Library. Oxford: Update Software.

\section{COMMENTARY}

The carefully done review by Gibson and colleagues shows that some but not all of the questions asked by spinal surgeons and their patients have been addressed in clinical trials. This review also emphasises the need to improve the standard of clinical trials in spinal surgery. Discectomy was used before the RCT design was developed, and one might have hoped for $>10$ RCTS studying outcomes of the evolving techniques. Some evidence exists of improvement in these studies over time. Only 1 RCT has compared discectomy with conservative treatment. 3 RCTs comparing conventional discectomy with the more recent microdiscectomy show no difference.

Surgery for degenerative lumbar spondylosis has been controversial for many years, with wide variations in practice. Despite this fact, clinical trials have only recently begun comparing spinal fusion with conservative programmes, and we do not yet know their outcomes. 9 trials have compared instrumented spinal fusion with noninstrumented fusion, which suggests that some concern exists about the value of the procedure. Resolving its effectiveness should have been the first question of researchers. However, surgical trials are seldom easy to design or conduct, and we surgeons have been generally slow to consider randomly allocating patients to new or alternative types of treatment.

This review provides some evidence to guide practice, although other important questions have not been addressed. It is worth reading in detail.

William J Gillespie, MBChB University of Otago Dunedin, New Zealand 